\title{
CALCULATION OF THE INDUCTANCE OF PLASMA COLUMN AT PF-1000 DEVICE WITH ASSUMED CURRENT DISTRIBUTION
}

\author{
JiRi KortaneK*, PAVEl Kubes
}

\begin{abstract}
CTU in Prague, FEE, Department of Physics, Technicka 2, 16627 Prague 6, Czech Republic
\end{abstract}
* corresponding author: kortanek@email.cz

\begin{abstract}
Processed data is taken from the D-D fusion reaction experiments on PF-1000 at IFPILM Warsaw, operating with $2 \mathrm{MA}, 10^{11}$ neutron yield, using interferometry, temporal resolved neutron diagnostics, magnetic probe diagnostics and X-ray diagnostics. The inductance is calculated under two different circumstances: with the known distribution function determined by the magnetic probe signal, and with the border of the plasma column determined from interferograms using Matlab. The inductance of the whole column is calculated using the formula for the inductance of a coaxial cylinder.
\end{abstract}

KEYWORDS: nuclear fusion, plasma focus, pinch, inductance, diagnostics, Matlab.

\section{INTRODUCTION}

Interferometry is a method for visualizing plasma and for obtaining some information about its structure and density. It has been used to measure plasma with density above $10^{23} \mathrm{~m}^{-3}$ in various PF devices [9, 1].

The PF-1000 plasma focus device, located at IPPLM in Warsaw, has recently been operating with $2 \mathrm{MA}$ with $10^{11}$ neutron yield [7]. Interferometry, neutron diagnostics, hard X-ray diagnostics, and magnetic probes diagnostics [5] are used, along with measurements of current, its time-derivative and voltage with nanosecond resolution. BC408 scintillators and Hamatsu photomultipliers are used for temporal resolved hard X-ray and neutron diagnostics. They are positioned side-on $(7 \mathrm{~m})$ and in the upstream direction $(7 \div 84 \mathrm{~m})$. Counters with activated silver are used for total neutron yield estimation [4.

The magnetic probes are induction-type probes customized specifically for the PF-1000 device. They are located at the face of the anode, $1.3 \mathrm{~cm}$ and $4 \mathrm{~cm}$ from the axis, as shown in Fig. 11 They register the temporal derivative of the azimuthal magnetic field at the anode front, and this enables the current density to be determined. An example of a signal from the probe in $1.3 \mathrm{~cm}$ is shown in Fig. 2 The $y$ axis was adjusted to have a maximum value of 1 for better evaluation. Its scale is therefore arbitrary. The value $x=0$ represents the time of the maximum soft X-ray signal. This applies to all figures in this paper, unless otherwise stated.

The interferometer at PF-1000 was designed and installed at IPPLM in Warsaw. The beam from the Nd:YLF EKSPLA SL300 series laser, operating on second harmonic wavelength $527 \mathrm{~nm}$ with pulse length below $1 \mathrm{~ns}$, is divided into 16 mutually delayed beams. The time step between the beams alternates between $10 \mathrm{~ns}$ and $20 \mathrm{~ns}$ [10. Interferograms are created which show us the time-evolution of the pinch

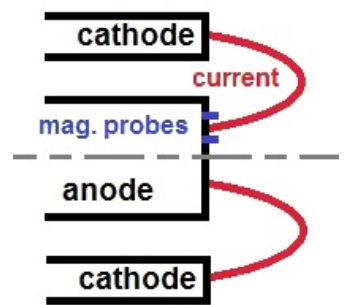

Figure 1. Schematic of the experimental setup.

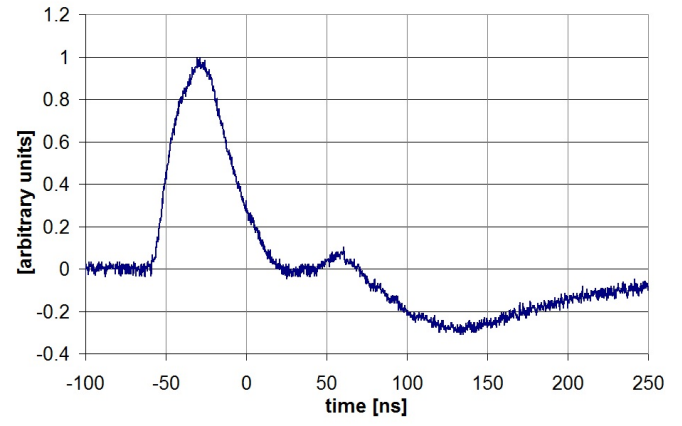

FiguRE 2. Signal from the magnetic probe in radial position $1.3 \mathrm{~cm}$ from the axis of the anode. Shot No. 9367.

over the course of $220 \mathrm{~ns}$ in one shot [10]. With these, we can correlate the time-evolution of the pinch dynamics with the neutron and X-ray emission [8, 6].

Interferometry is a path-integrated technique, so a method based on Abel transformation is used for axial symmetry [2]. More accurate unsymmetrical densitograms are then created using a method presented in [3]. An example of a densitogram of this type is shown in Fig. 3

The aim of this work is to use two different calculations of the inductance of the plasma column for two temporal intervals. The first calculation is based on detecting the borders of the plasma after the implosion, 


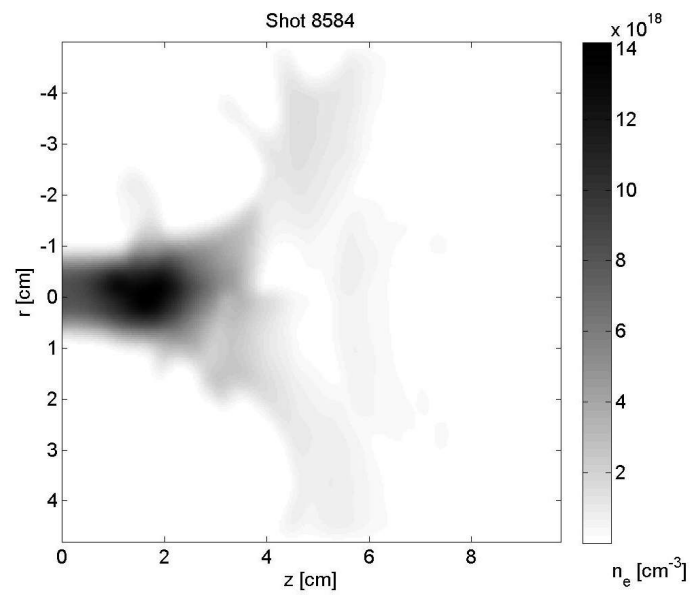

Figure 3. Asymmetrical densitogram of shot No. 8584.

and the second calculation is based on estimating the current distribution from the magnetic probe data during the implosion.

\section{Calculations}

\subsection{Current flows Along the border OF THE DENSE PLASMA COLUMN}

For the calculation of the inductance, we presume that after the implosion the mean value of the current flows along a thin layer on the surface of the dense plasma column.

The application detects the borders of the plasma by finding the threshold value of the density. The search algorithm searches for the first and last pixel with this threshold value in each column of pixels. These two ivalues are designated the upper and lower border of the plasma for the current pixel column. With known position of the borders, we can determine the radius $r$ of the plasma column. The formula for determining the threshold density was

$$
D=D_{\operatorname{lm}}\left[k_{1}+k_{2}\left(1-D_{\operatorname{lm}} / D_{\max }\right)\right] \quad\left[\mathrm{cm}^{-3}\right],
$$

where $D_{\operatorname{lm}}$ is the maximum density in the current column of pixels, $D_{\max }$ is the maximum density in the whole shot, and $k_{1}$ and $k_{2}$ are constants. These constants are set for the borders to follow the places where we see a sharp rise or drop in plasma density. We need to set them experimentally for each shot, because each shot has its own special density ratios. For shot 9013 , the values were determined $k_{1}=0.23$ and $k_{2}=0.65$. The detected borders of the plasma column are shown in Fig. 4.

The formula for the inductance $L$ of a coaxial cylinder is used,

$$
\sum_{1}^{n} \Delta L=\sum_{1}^{n}\left(\Delta l \frac{\mu}{2 \pi} \ln \frac{R}{r}\right) \quad[H]
$$

with length of the pixel in meters $\Delta l, \mu$ is the magnetic constant $\left(4 \pi \times 10^{-7} \mathrm{H} / \mathrm{m}\right), r$ is the inner radius determined by the detected borders of the plasma,

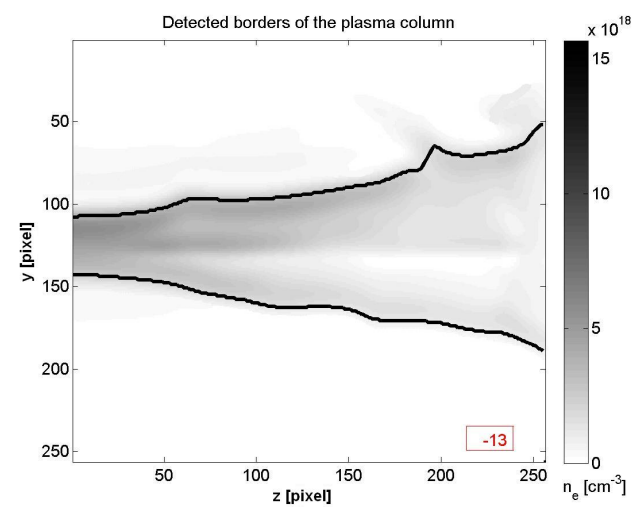

Figure 4. Detected borders in the densitogram of shot No. 9013, $t=-13$ ns (relative to the time of the maximum pinch).

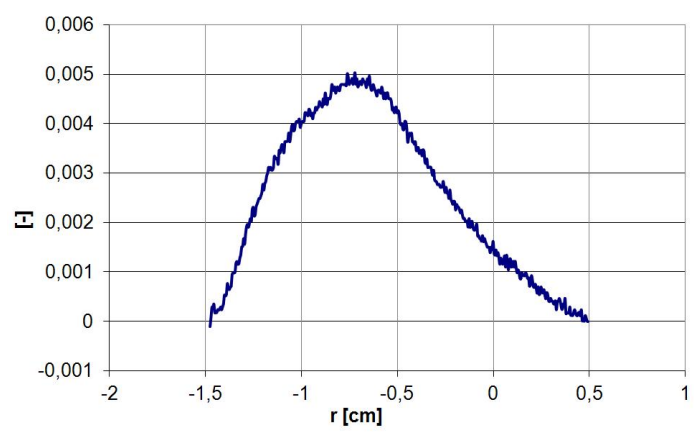

FiguRE 5. Distribution function of the current flow at the time of pinch for shot No. $9367 ; x=0$ represents the position of the magnetic probe, positive values are towards the center of the anode.

$R$ is the radius of the outer electrodes $(15 \mathrm{~cm})$, and $n$ is the number of the pixel columns in the current picture.

The application calculates the inductance of each column of pixels and adds them up to get the inductance of the whole column. When this process is used for every densitogram in the shot, the time resolved inductance is obtained.

\subsection{Current Flows Distributed}

The magnetic probe signal was registered near the anode face in temporal scale dependence. To obtain the radial distribution of the current during the implosion, we use the formula

$$
s=v t \quad[\mathrm{~m}]
$$

where $s$ is length, $v$ is implosion velocity and $t$ represents time. We used implosion velocity $2.5 \times 10^{5} \mathrm{~m} / \mathrm{s}$ with relative uncertainty of $20 \%$ calculated from the positional differences of the plasma border at the same $z$-coordinates in the succeeding interferograms.

The signal is then truncated to contain only the region of the first peak, where the magnetic probe is not yet damaged by the high-temperature plasma. The values of this signal are adjusted so that the sum 


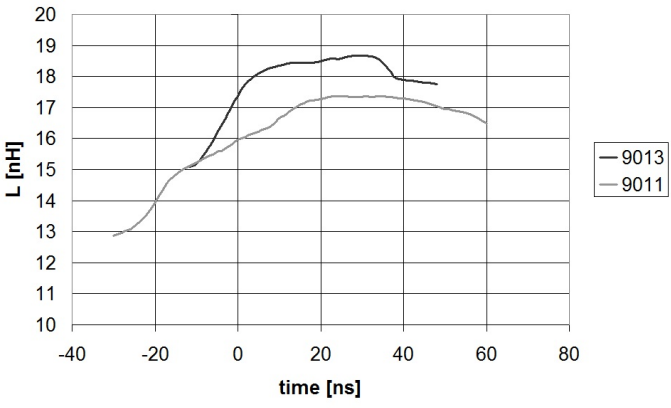

FiguRE 6. Inductance of shots No. 9011 and No. 9013 using plasma border detection.

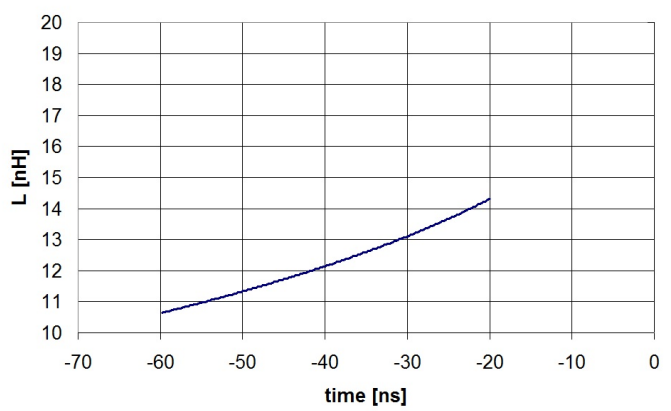

Figure 7. Inductance of shot No. 9367 using the current distribution.

of the signal equals 1 . Thus it does not have physical units. The acquired function, shown in Fig. 5, is used as a distribution function of the current flow.

We assume the same radial distribution of the current for all axial positions toward the boundary.

With the known distribution function of the current, Eq. 2 can be used again. In this case, $r$ is the current layer diameter and $R$ is again the radius of the electrodes $(15 \mathrm{~cm})$.

The inductance is calculated for each current layer, i.e. row of pixels. It is multiplied by the corresponding value of the distribution function, and added us up to get the inductance of the whole plasma column. The time-resolved inductance is acquired by applying this process for every nanosecond in the shot.

\section{Results}

The results of the border detection method are shown in Fig. 6 for shots No. 9011 and No. 9013. A similar slow increase in inductance from $15 \mathrm{nH}$ at $-10 \mathrm{~ns}$ to $18 \mathrm{nH}$ at $40 \div 50 \mathrm{~ns}$ was also observed in other shots. Later the inductance slowly decreases.

The result of the calculation based on the current distribution measured by the magnetic probes for shot No. 9367 is shown in Fig. 7. The differences between the results for other shots were below an uncertainty value of $20 \%$.

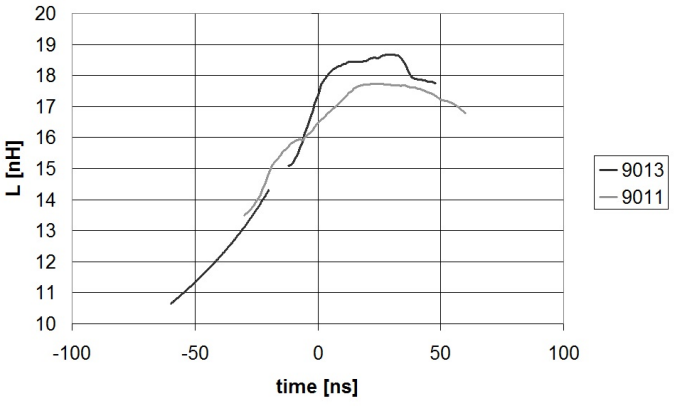

Figure 8. Comparison of the resulting inductances for two calculations for shots No. 9011 and No. 9013.

\section{Conclusions}

The inductance of the plasma column has been estimated from shots No. 9011, 9013 and 9367.

Each of the calculations can be used only at certain times, specified by the limitations of the diagnostic methods.

This plasma border detection method can be used only when we have interferometric data for the times after the implosion phase. For shot No. 9011, the time was from $-30 \mathrm{~ns}$ to $60 \mathrm{~ns}$, and for shot No. 9013 the time was from $-12 \mathrm{~ns}$ to $48 \mathrm{~ns}$.

The current distribution can be used only during the time of the implosion. The distribution is similar for different shots, so the conclusions about the temporal evolution of the inductance can be generalized with uncertainty of $20 \%$.

According to the magnetic probe method, most of the current flows about $1 \mathrm{~cm}$ above the border of the dense plasma column during the implosion phase falls towards the plasma column during the stagnation phase, and could even sink into the plasma later during the expansion.

The composition of the two calculations, see Fig. 8 shows that there is a connection between them in the intersection region.

This shows that the assumption made in the plasma border method - that the mean value of the current flows along a thin layer of the plasma column - can be taken as valid, at least after the implosion phase.

\section{ACKNOWLEDGEMENTS}

This research has been supported by research program No. LA08024 "Research in the Frame of the International Center for Dense Magnetized Plasmas", by research program No. ME09087 "Research of Plasma of Fast Z-Pinches" of the Ministry of Education, Youth and Sport of the Czech Republic, and by the GACR grants No. P205/12/0454 "Effective Production of Fusion Neutrons in Z-Pinch and Laser Produced Plasma", grant CR IAEA 14817 "Research of D-D fusion reactions at the CTU in Prague", grant CTU SGS 10-2660-OHK3-3T-13, and by grant No. 0661/B/H03/2011/40, supported in part by the National Science Centre. 


\section{REFERENCES}

[1] A. Bernard, A. Coudeville, et al. Experimental studies of the plasma focus and evidence for nonthermal processes. Phys Fluids 18(2):180-194, 1975.

[2] A. Kasperczuk, T. Pisarczyk. Application of automated interferometric system for investigation of the behaviour of a laser-produced plasma in strong external magnetic fields. Opt Appl 31(3):571-597, 2001.

[3] J. Kortanek, et al. Matlab applications for processing of interferograms and oscilograms from PF-1000 experiments. In Tenth Kudowa Summer School. IPPLM, 2011.

[4] J. Krasa, M. Kralik, et al. Anisotropy of the emission of DD-fusion neutrons impressed by the plasma-focus vessel. Plasma Physics and Controlled Fusion 50(12):125006, 2008.

[5] V. Krauz, K. Mitrofanov, et al. Experimental study of the structure of the plasma-current sheath on the PF-1000 facility. Plasma Physics and Controlled Fusion 54:025010, 2012.
[6] P. Kubes, et al. Evolution of the gass-puff Z-pinch column. IEEE Transactions on Plasma Science 26(4):1113-1118, 1998.

[7] P. Kubes, et al. Spontaneus transformation in the pinched column of the plasma focus. IEEE Transactions on Plasma Science 39(1):562-568, 2011.

[8] P. Kubes, M. Paduch, et al. Interferometric study of pinch phase in plasma-focus discharge at the time of neutron production. IEEE TPS 37(11):2191-2196, 2009.

[9] P. Schmidt, P. Kubes, et al. Neutron emission characteristics of pinched dense magnetized plasma. IEEE Trans Plasma Sci 34(5):2363-2367, 2006.

[10] E. Zielinska, M. Paduch, et al. Sixteen-frame interferometer for a study of a pinch dynamics in PF-1000 device. Contrib Plasma Phys 51(2-3):279-283, 2011. 